\title{
EL TALLER REALEJO, UN REFERENTE DEL GRABADO EN GRANADA
}

\section{León Moreno García}

\section{leonmoreno76@yahoo.com}

Universidad de Granada. Dpto. Historia del Arte, Granada, España.

\section{Resumen}

En este trabajo se realiza un repaso histórico a la trayectoria del Taller de Grabado Experimental EI Realejo, de Granada.

Se exponen sus principales contribuciones artísticas así como sus representantes más destacados. Se aportan elementos para conocer la importancia de este Taller en la ciudad de la Alhambra, integrado por un grupo de creadores que provenían de otro colectivo artístico llamado "Acción 25" fundado anteriormente.

Junto con las ediciones de grabados en suscripciones y carpetas, el Taller Experimental de Grabado El Realejo también vino realizando una destacable tarea de difusión, mediante la organización de exposiciones de sus distintos componentes. De esta importante labor, se indican igualmente casi cuatro decenas de las más importantes.

La presencia de su obra en museos y colecciones nacionales e internacionales evidencian la relevancia de su producción en el contexto de la Historia del Arte. Para conocer mejor este Taller se aportan elementos contextuales que explican la realidad del mismo como un proyecto abierto a la creación y ejemplo de labor artística en la que se juntan sensibilidades muy distintas, para, de esta forma, todos ellos formar un grupo de autores a través de los cuales puede apreciarse el ímpetu y la diversidad del grabado vigente actualmente en Granada, una urbe con una tradición calcográfica bastante considerable que comenzó en el siglo XV.

\section{Palabras clave}

Historia del Arte, Taller, Grabado, Realejo, Granada.

\section{Repaso Histórico a la Trayectoria del Taller de Grabado Experimental El Realejo.}

El Taller de Grabado ${ }^{1}$ Experimental El Realejo se constituye en la ciudad de Granada en el mes de septiembre de 1985 y, en un principio, estaba integrado por un grupo de creadores que provenían de otro colectivo artístico llamado "Acción 25" fundado anteriormente.

Así, de los artistas fundadores del Taller Realejo continuaron en el grupo bastantes años como miembros activos tan sólo algunos, entre éstos Dolores Montijano, Rosario García Morales, Teiko Mori o Eduardo Fresneda, otros como Manini Ximénez de Cisneros, Juan Orozco, Carmen Sicre, Manuel Vela o Jesús Conde permanecieron escaso tiempo en él ya

1 "Hoy, pues, el grabado no es siervo de las Artes Gráficas, sino expresión directa y singular del propio artista. Al menos ha retornado a su condición primera y categórica". Izquierdo, Francisco: Grabadores Granadinos. Editorial Marsiega, Madrid, 1974. Pág. 60. 
que se dedicaron, al poco de fundado, más a la actividad de sus estudios de pintura o talleres personales de grabado; y en fecha inmediatamente posterior a su fundación se incorporaron Cayetano Aníbal, Juan Manuel Brazam y Manuel del Moral, aunque por escaso tiempo los dos últimos citados. Igualmente se integró en el grupo Julio Espadafor, pero no llega a incorporarse plenamente debido a su temprana desaparición. Más adelante entran en el Taller Luís Orihuela, Francisco Izquierdo, Juan Carlos Lazuen, María José de Córdoba y ya en etapas posteriores, Julián Amores, Carlos Villalobos, Tremedad Gnecco, José Manuel Peña, Araceli de La Chica y Armando Salas. Debemos reseñar y es un hecho constatable que muchos de los grabadores que han pasado por el Realejo se iniciaron en el Taller de la Fundación Rodríguez Acosta bajo las enseñanzas de José García de Lomas, ya desaparecido y que también fue durante un tiempo miembro significado.

Por otra parte, y siguiendo con este breve repaso sobre la trayectoria del Taller de Grabado Realejo hemos de decir que en un principio el domicilio social del Taller estuvo desde la fecha de su fundación en la Calle Molinos ํ‥49, bajo derecha, donde se instaló este taller de grabado, pero a la postre, en el mes de septiembre de 2002 se traslada a la calle San Jacinto $n \div .20$ en el bajo derecha, donde se encuentra actualmente.

Así, y continuando con este breve repaso histórico, constatamos que en el mes de julio de 1987 se inscribe legalmente como "Taller Experimental de Artes Plásticas", aprobados sus estatutos por la Delegación del Gobierno de Granada con los fines siguientes:

-Defensa y protección del Patrimonio Histórico-Artístico.

-Fomentar el respeto y protección de todo aquello que constituya nuestra cultura.

-Organizar una constante labor formativa de promoción cultural. -La investigación y experimentación de nuevas técnicas en las artes plásticas.

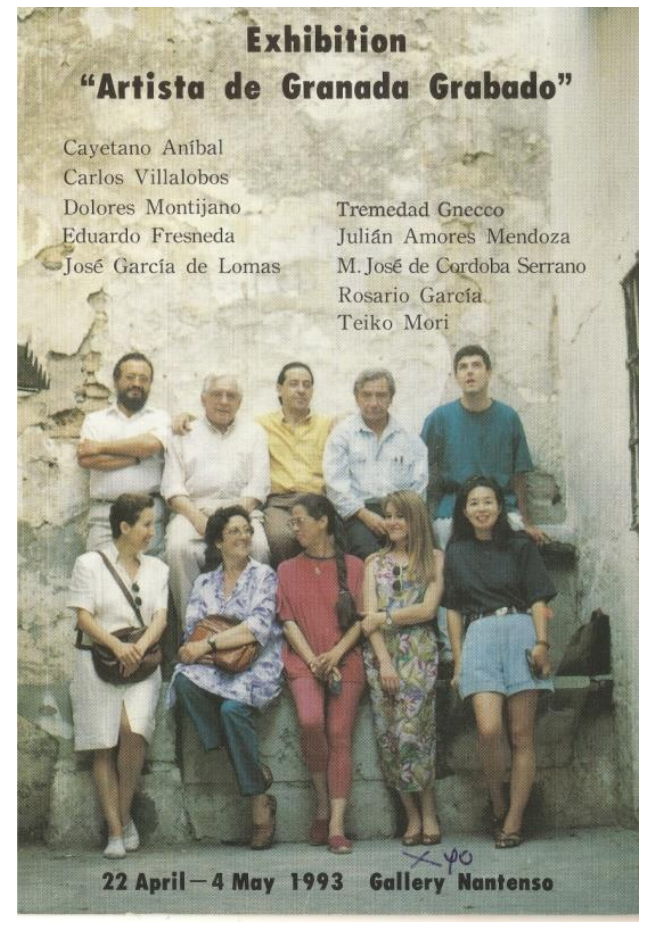

Ilustración 1. Tarjeta Postal Exposición Grabadores en Granada. Natenso Gallery. Kobe Shi. Japón. Abril de 1993. 
De esta forma, con las altas y bajas que se han producido durante los años de su existencia, el Taller Realejo en la actualidad lo componen los siguientes miembros: Rosario García Morales, Eduardo Fresneda, Cayetano Aníbal, Julián Amores, Carlos Villalobos, Tremedad Gnecco y Araceli de La Chica. De esta manera podemos atestiguar, sin lugar a dudas, que el grabado en Granada tiene un referente de privilegio con la labor que viene realizando desde hace ya tiempo el Taller Experimental de Grabado El Realejo.

Así, tras las experiencias que se llevaron a cabo en el Taller de la Fundación Rodríguez Acosta en los años setenta, bajo la dirección de José García de Lomas, y otras iniciativas de diferentes grupos artísticos como "Aldar" o "Acción 25" a principios de los ochenta, un conjunto de creadores, en su mayoría partícipes en aquellas empresas artísticas, llegaron al acuerdo de poner en marcha un taller, para el que recibieron ayuda de la Junta de Andalucía, que fue invertida en las infraestructuras necesarias.

De este modo, en el verano de 1985 se hacía realidad este proyecto que tenía como objetivo llevar a cabo un trabajo de búsqueda y de investigación técnica, sin limitaciones a la incorporación de nuevos procedimientos y lenguajes.

Como ya hemos mencionado anteriormente; enumeramos pero ahora con más detalle el núcleo fundador, integrado por los artistas Eduardo Fresneda, Rosario García Morales, Dolores Montijano, Juan Orozco, Jesús Vela, Carmen Sicre, Jesús Conde, Teiko Mori, Gilton Bastos, Manuel Pertíñez, José Antonio Hernández y Manini Ximénez de Cisneros, que recibió sucesivas incorporaciones y así fueron integrándose en él otros creadores tales como Cayetano Aníbal, Juan Manuel Brazam o Manuel del Moral. Más tarde lo harían Ana Beveraggi, María José de Córdoba, Luís Orihuela, Jesús Pertíñez y Juan Carlos Lazuén, hacia finales de los años ochenta se suman Julián Amores, Francisco Izquierdo y Carlos Villalobos, en los noventa, lo hacen el maestro José García de Lomas, Tremedad Gnecco y José Manuel Peña.

Por tanto, a lo largo de todos estos años, al margen de las fluctuaciones de nómina, muchos han sido los trabajos y exposiciones del colectivo y su participación en la vida cultural granadina queda reflejada en la edición de numerosas carpetas y suscripciones de taller, tales como "Loxa" (1986), con textos del poeta Juan de Loxa o "Cinco versiones gráficas de Salobreña" (1989), con escritos de José García Ladrón de Guevara y del dramaturgo José Martín Recuerda, además de otras ediciones como "Viento del Sur" (1990), en homenaje a Federico García Lorca, con poemas de Javier Egea, Luís García Montero, Antonio Jiménez Millán, Luís Muñoz y Álvaro Salvador y un preliminar de Antonio Sánchez Trigueros; "El Olvido y la Memoria" (1990), con escritos de Muñoz Molina, Tomás Calvo y Virginia Ruiz; "El curso de los Meses" (1995), con prosas poéticas de Francisco Izquierdo; "Las Rutas de AlAndalus" (1995), relacionada con el programa del Legado Andalusí y precedida de una presentación a cargo del poeta Rafael Guillén.

\section{Contribuciones artísticas más reseñables del Taller de Grabado El Realejo.}

Respecto a las exposiciones del grupo, constatamos que no han cesado a lo largo de todos estos años, desde la primera muestra colectiva "Homenaje a Julio Espadafor" en el año 1986 y hasta el momento, se han dado a conocer los trabajos del taller en numerosas ocasiones tanto en Granada en diversas salas e instituciones, como en otras ciudades españolas, tales como Alicante, Valladolid, Palencia, Soria, Ávila, Marbella, Madrid, Ceuta, etc., así como extranjeras, Santo Domingo, San Juan de Puerto Rico, etc.

Por tanto, comprobamos que el Taller es un proyecto abierto a la creación y ejemplo de labor artística en la que se juntan sensibilidades muy distintas, y, de esta forma, todos ellos forman un grupo de autores a través de los cuales puede apreciarse el ímpetu y la 
diversidad del grabado vigente actualmente en Granada, una urbe con una tradición calcográfica bastante considerable que comenzó en el siglo XV.

"Esa es la Andalucía compleja y múltiple que se expresa en la obra de los artistas que constituyen el "Taller Experimental de Grabado El Realejo". Andalucía de zetas, eses y ces, de anfibologías y metáforas, de hipérboles exageradas. Andalucía de rojos y de blancos, pero también de colores indescifrables, Andalucía de trazos únicos, Andalucía de densas metáforas. Andalucía de ocho artistas, ocho: Julián Amores, Cayetano Aníbal, Araceli de la Chica, Eduardo Fresneda, Tremedad Gnecco, Francisco Izquierdo, Rosario García Morales, Carlos Villalobos, ocho grabadores para una metáfora: "en un trono de sangre, anda-luz de levante y poniente, por la ventana, muros de luz y sombra, contraventanas del pueblo, soleá, pseudotauromaquia" que da título a las obras que se exponen en Contemporánea como si de un mapa de ocho provincias se tratara". ${ }^{2}$
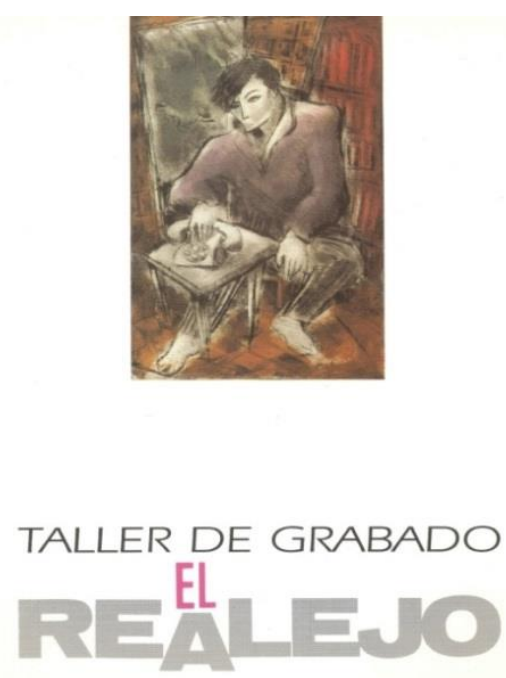

GRANADA

Ilustración 2. Portada del Catálogo de la Exposición Taller de Grabado Experimental El Realejo con el grabado "Sin Línea" (1993) de Cayetano Aníbal. Catálogo n. 62 de la Serie "Artistas Plásticos". Fundación Caja de Granada.

También podemos observar que uno de los puntos fuertes que ofrece este taller de grabado se cifra en la multiplicidad de técnicas y estilos de los que se valen para crear las láminas, así, este dato nos da información acerca de la multiplicidad de temáticas que sustentan las láminas, y además cada uno aporta su forma diferente de concebir la estampa.

"Aventura abierta a la creación y ejemplo de labor en la que se juntan sensibilidades muy distintas, el grupo lo forman, en la actualidad, los artistas: Julián Amores, Cayetano Aníbal, María José de Córdoba, Eduardo Fresneda, Rosario García Morales, Tremedad Gnecco, Francisco Izquierdo, Dolores Montijano, Teiko Mori, Luis Orihuela, José Manuel

2 Vellido, Juan. “De la Tierra Múltiple”. Catálogo AndaluzScias. Taller Experimental de Grabado El Realejo. Editorial Contemporánea Centro de Arte, Granada, 2002. Pág.3. 
Peña y Carlos Villalobos, doce autores a través de los cuales puede constatarse la versatilidad y la fuerza de la estampa actual en Granada, una ciudad con un pasado calcográfico de primer orden, desde finales del siglo XV. Uno de los atractivos que ofrece este colectivo de artistas se cifra en la variedad de estilos, técnicas y procedimientos utilizados a la hora de conformar las planchas. Este hecho da idea de la diversidad de atmósferas, mundos o preocupaciones temáticas que nutren las láminas."

De esta manera, aseveramos, sin lugar a dudas que con el Taller de Grabado Experimental El Realejo, la vanguardia del arte granadino alcanza un nivel tanto en lo plástico, en lo técnico, como en lo sensitivo y en los contenidos, que nos informa de la vitalidad y de la madurez del ejercicio calcográfico en la ciudad.

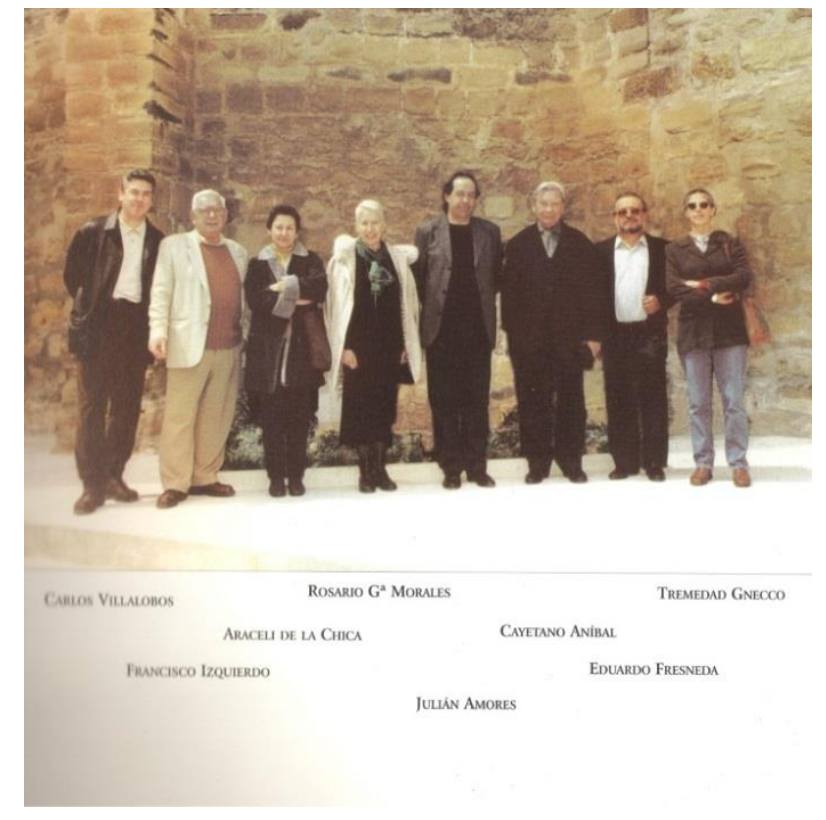

Ilustración 3. Componentes del Taller Realejo en el año 2002. Fotografía incluida en el Catálogo de la Exposición "AndaluzScias".

"Rastrear, pues, el arte granadino para hallar una pista o documento sobre grabadores es una aventura penosa y desalentadora. Ya lo ha sido cuando se ha tratado de fijar un panorama total o parcial de la pintura, reconociendo que la información en este campo es más generosa. Y así nos lo confirma aquel noble intento de Antonio Aróstegui con "Arte granadino actual", libro que, hace ahora doce años, se quedó incomprensiblemente en embarazo de imprenta. En ese caminar por el arte granadino a veces tropieza el investigador con el nombre de un pintor que realizó tal obra para tal lugar, siendo la noticia de la obra o del lugar mucho más amplía. Y nada más. Un nombre solitario del que jamás vuelve a saberse. A veces, se despacha una nómina de artistas como asistentes a tal exposición, sin ningún otro dato que el árido del nombre y el apellido o del apellido sólo. Otras se encuentran esos nombres en listas de suscriptores, de socios o de miembros de tertulias, liceos o publicaciones. "Fulano de Tal, pintor", y pare usted de contar. $Y$, en

3 Lupiáñez, José: “El grabado de vanguardia en Granada”. Catálogo Taller de grabado El Realejo. Serie Artistas Plásticos. Ed. Fundación Caja Granada, Granada, 1996. Págs. 2-3. 
muchos casos, aparecen como maestros de discípulos aventajados: "Mengano de Cual, discípulo de Zutano", pero resulta que es la primera y única de Zotano". ${ }^{4}$

Es un hecho evidente que el Taller Realejo se ha constituido verdaderamente en uno de los focos en los que se sustenta el futuro del grabado en Granada, ya que la mayor parte de sus experiencias han sido y serán un referente obligatorio para posteriores creaciones estéticas. Del mismo modo, sus integrantes se ha caracterizado siempre por su creatividad, igualmente han sido artistas fieles a su compromiso con el arte y con el tiempo en el que han trabajado, y han dado una lección de entrega y de veracidad que se comprueba fácilmente al contemplar su obra.

"Doce itinerarios, pues, doce nombres y doce formas distintas de concebir la estampa. Estimula saber que a través de ellos la vanguardia del arte granadino alcanza un nivel, en lo plástico, en lo técnico, en lo sensitivo, en los contenidos...que habla de la vitalidad y la madurez del ejercicio calcográfico entre nosotros. El Taller Experimental El Realejo es, sin lugar a dudas, uno de los centros que se asegura el porvenir del grabado, puesto que muchas de sus experiencias de hoy serán mañana el punto de partida obligatorio para futuras aventuras estéticas". 5

Por todos estos motivos, actualmente en Granada en el ámbito del grabado no puede dejar de citarse el Taller Experimental Realejo, además en pocos lugares pueden estar orgullosos de contar con grabadores que, no sólo rinden homenaje a la tradición, sino que anticipan, con sus experiencias, los caminos por donde habrán de discurrir necesariamente las nuevas incursiones gráficas.

\section{Ediciones de Grabados en Suscripciones y Carpetas.}

Entre las ediciones desarrolladas por el Taller Experimental de Grabado El Realejo destacamos las siguientes que enumeramos a continuación por orden cronológico:

En 1990 se editó por el Instituto García Lorca de Churriana la carpeta de 9 grabados "Viento del Sur" en tirada de 50 ejemplares más 9 de la A a la I y 9 de I a IX con diseño de Julián Amores, texto de presentación de Antonio Sánchez Trigueros y poemas de Javier Egea, Luís García Montero, Antonio Jiménez Millán, Luis Muñoz y Álvaro Salvador, y con grabados de Dolores Montijano, Rosario García Morales, Julián Amores, Cayetano Aníbal, Teiko Mori, Eduardo Fresneda, Francisco Izquierdo y Carlos Villalobos.

En 1990-1991 sale editada una colección de 10 grabados en suscripción con tirada de 50 ejemplares más 10 de la $\mathrm{A}$ a la $\mathrm{J}$ y originales de Dolores Montijano, Rosario García Morales, Eduardo Fresneda, Cayetano Aníbal, José García de Lomas, Teiko Mori, Francisco Izquierdo, Julián Amores, María José de Córdoba y Carlos Villalobos.

En 1991, el taller participó, invitado, en la edición de la carpeta "El arte de grabar en Granada" con tirada de 75 ejemplares, con originales de Julián Amores, Cayetano Aníbal, María José de Córdoba, Eduardo Fresneda, Rosario García Morales, Tremedad Gnecco, Francisco Izquierdo, Dolores Montijano y Carlos Villalobos. Durante 1991-1992 se puso en marcha otra suscripción de 10 grabados con edición de 50 ejemplares más de la A a la J. Los autores fueron Rosario García Morales, Dolores Montijano, Francisco Izquierdo, Cayetano Aníbal, Eduardo Fresneda, Teiko Mori, Julián Amores, Carlos Villalobos, Tremedad Gnecco y María José de Córdoba.

4 Izquierdo, Francisco, op. cit., Pág. 6.

5 Lupiáñez, José, op. cit., Pág. 10. 
Ilustración 4. Portada del Catálogo "AndaluzScias". Diseño realizado por Cayetano Aníbal (2002).

Con los mismos autores, en 1993 se editó otra colección de 10 grabados en suscripción con tirada en número de 50 ejemplares y otros de la A a la J y de I a X. En 1994 se edita una carpeta de 12 grabados "El Curso de los meses" con edición de 75 ejemplares más otros 12 de la $A$ a la L, y con textos de Francisco Izquierdo, el diseño estuvo a cargo de Julián Amores y Cayetano Aníbal, y los grabados fueron realizados por Julián Amores, Rosario García Morales, Dolores Montijano, Francisco Izquierdo, Teiko Mori, Luís Orihuela, José Manuel Peña, Carlos Villalobos,

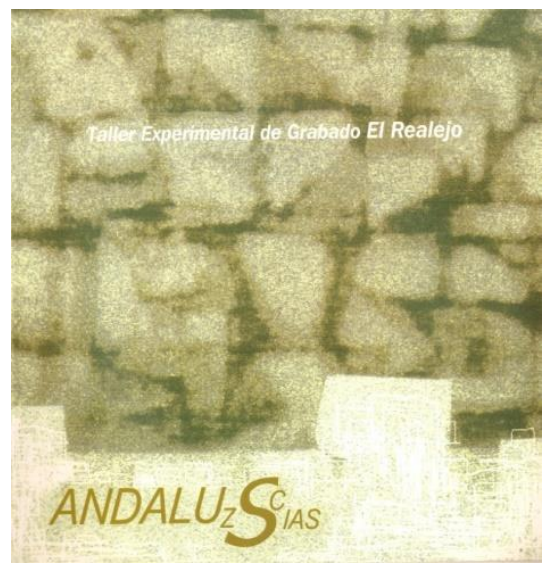
Cayetano Aníbal, Eduardo Fresneda, María José de Córdoba y Tremedad Gnecco.

En 1995, por encargo de El Legado Andalusí, es editada la carpeta de 12 estampas "Las Rutas de Al-Andalus" con edición de 70 ejemplares más 12 de la $A$ a la $L$ y 10 de I a X, con diseño de Cayetano Aníbal, texto de presentación de Rafael Guillén y selección de textos arábigo-andaluces de Mauricio Pastor y Concepción Castillo, y con los grabados originales de Cayetano Aníbal, Tremedad Gnecco, José Manuel Peña, Eduardo Fresneda, Julián Amores, Francisco Izquierdo, Dolores Montijano, Carlos Villalobos, Luís Orihuela, Rosario García Morales, Teiko Mori y María José de Córdoba.

En 1996 se edita la colección de 12 estampas, "El Realejo según El Realejo", como homenaje al barrio en que se ubicaba el Taller. La edición constaba de 50 ejemplares más 15 de la $\mathrm{A}$ a la $\tilde{\mathrm{N}}$, Cayetano Aníbal realizó el diseño, con textos escritos de José García Ladrón de Guevara, y participando los grabadores Julián Amores, Cayetano Aníbal, María José de Córdoba, Eduardo Fresneda, Rosario García Morales, Tremedad Gnecco, Francisco Izquierdo, Dolores Montijano, Teiko Mori, Luís Orihuela, José Manuel Peña y Carlos Villalobos.

En 1997, por iniciativa de la Delegación Provincial de Cultura de la Junta de Andalucía, se editó la carpeta de 8 estampas "Retablillo Lorquiano" sobre el teatro breve de Federico García Lorca, la edición constaba de 50 ejemplares más 8 de la $\mathrm{A}$ a la $\mathrm{H}$, con diseño de Cayetano Aníbal, textos de Enrique Moratalla y Antonio Sánchez Trigueros, y grabados de Julián Amores, Cayetano Aníbal, Eduardo Fresneda, Rosario García Morales, Tremedad Gnecco, Francisco Izquierdo, José Manuel Peña y Carlos Villalobos.

De 1999 es el libro-carpeta "Tiempo de deseo" con 14 grabados, editado por el propio taller, con una edición de 50 ejemplares más 7 de la A a la G y otros 7 de I a VII. El diseño corrió a cargo de Cayetano Aníbal, y se utilizó como texto de introducción el "Ars Amandi" de Ovidio, con las planchas originales de Julián Amores, Cayetano Aníbal, Eduardo Fresneda, Rosario García Morales, Tremedad Gnecco, Francisco Izquierdo y Carlos Villalobos.

"Emociona asistir a esta eclosión creativa, a este sueño compartido, que los artistas han hecho posible, porque han sabido ser fieles a su compromiso con el arte y con el tiempo en el que discurren sus biografías. Ingente lección de entrega y de verdad que estamos obligados a valorar todos los que somos sus destinatarios. Pocas veces se dan estas circunstancias. El hoy de la estampa en Granada no puede permitirse desdeñar este inmenso legado, ni desatender este estimulante recorrido que es ya una conquista de las que nos beneficiaremos tantos y de la que habrán de nutrirse las próximas generaciones. Pocas ciudades pueden ufanarse de contar con creadores que, no sólo rinden homenaje a 
la tradición, sino que anticipan, con su experiencia, los caminos por donde habrán de discurrir necesariamente las nuevas tentativas. Es cuestión de justicia y de conciencia. ${ }^{16}$

En 2000 participan todos los miembros del taller en la carpeta "Estampas para la Paz", editada por el Instituto de la Paz y los Conflictos de la Universidad de Granada.

La edición constaba de 75 ejemplares más 23 de la $\mathrm{A}$ a la $\mathrm{V}$, con diseños de Cayetano Aníbal, texto de presentación de Ignacio Henares e incluyó un poema de Antonio Carvajal, apareciendo los grabados de Cayetano Aníbal, Eduardo Fresneda, Rosario García Morales, Tremedad Gnecco, Francisco Izquierdo, Carlos Villalobos y una serigrafía de Julián Amores.

En 2000-2001 se edita una colección de 8 grabados en suscripción con tirada de 48 ejemplares más 8 de la $\mathrm{A}$ a la $\mathrm{H}$ y 8 de I a VIII, con unas matrices originales de Rosario García Morales, Eduardo Fresneda, Cayetano Aníbal, Francisco Izquierdo, Julián Amores, Carlos Villalobos, Tremedad Gnecco y Araceli de La Chica.

Y ya en 2003 se edita por el A.M.P.A. del Colegio de Educación Especial San Rafael de Granada, la carpeta de grabados "Cuentos y Sueños" con 50 ejemplares más 9 de la A a la L. La realización de las matrices se realizó desinteresadamente por los componentes del Taller Realejo, haciendo el diseño Carlos Villalobos, y con grabados originales de Julián Amores, Cayetano Aníbal, Araceli de La Chica, Eduardo Fresneda, Rosario García Morales, Tremedad Gnecco, Francisco Izquierdo, Armando Salas y Carlos Villalobos.

La edición de 2003 es la última realizada por este taller hasta la actualidad. De esta forma, componen un total de once ediciones que nos constatan la fecundidad artística de este colectivo de creadores.

"De esa Andalucía ha de quedarnos el recelo del duende surreal y esperpéntico en la sutilísima y lúcida "Pseudotauromaquia" de Julián Amores: el largo camino a los dos lados de "Contraventanas del pueblo" de un Cayetano Aníbal sugerente y medido en sus matices y tonos: los geométricos espacios de los "Muros de luz y sombra" de una Araceli de la Chica que ha delimitado lugar y concepto en un singular juego de volúmenes y sombras: los fondos sin fondo de "La ventana" Ilena historia con la que Eduardo Fresneda conduce su singular visión del tiempo que transcurre: el "Levante-poniente" con el que Tremedad Gnecco insinúa relieves geográficos y humanos, como un símil entre la piel y el manto dúctil de las montañas los valles: la "Soleá" rota, quebrada, de Francisco Izquierdo, con dos paisajes sentidos, uno de fondo, otro en primer plano, uno lleno de arrugas, otro de casas encaladas: el "Andaluz" pasional, en todos los matices del rojo, en el profundo amarillo del fuego, de esa luz que como alas de mariposa expande Rosario García Morales: y el "Trono de sangre" de la Andalucía rural e inequívoca, pletórica de símbolos, de Carlos Villalobos".

\section{Exposiciones de Grabado del Taller Realejo.}

Junto con las ediciones de grabados en suscripciones y carpetas el Taller Experimental de Grabado El Realejo también ha realizado una importante labor de difusión organizando exposiciones ${ }^{8}$ de sus distintos componentes.

\footnotetext{
6 Lupiáñez, José, op. cit., Pág. 10.

$7 \quad$ Vellido, Juan, op. cit., Pág.4.

8 "Por lo que se refiere a las exposiciones de grupo, puede decirse que no han cesado a lo largo de todos estos años. Desde la muestra colectiva "Homenaje a Julio Espadafor" (1986) hasta el presente se han dado a conocer los trabajos del Taller en numerosas ocasiones, tanto en Granada, en diversas salas e instituciones, como en otras ciudades españolas (Alicante, Valladolid, Palencia, Soria, Ávila, Marbella, Madrid, Ceuta, etc.) y
} 
Reseñamos a continuación las más significativas:

1986. Diciembre. Exposición Homenaje a Julio Espadafor. Escuela de Arte. Granada.

1987. Abril. Grabadores del Taller Experimental Realejo. Sala de Arte Caja Provincial. Loja. Granada.

1988. Agosto. Doce Grabadores. Peña Cultural. Las Gabias. Granada.

1990. Noviembre, Diciembre. Gráfica en Granada. Sala Municipal de Arte. Alicante.

1991. Enero, Febrero. Gráfica. Galería Cartel. Granada.

1992. Enero, Marzo. Participa en El arte de grabar en Granada. Valladolid, Palencia, Soria, Ávila.

1992. Abril. Invitado a participar en Granada ante el 92. Palacio de la Madraza. Granada.

1992. Mayo. Participa en la XVIII Bienal de Artes Audiovisuales. Galería de Arte Moderno. Santo Domingo. República Dominicana.

1993. Febrero. Invitados en Grabadores granadinos en el 92. Casa de Andalucía. Puerto Rico.

1993. Abril. Grabadores en Granada. Natenso Gallery. Kobe Shi. Japón.

1993. Mayo. Mirando a Miró. Galería Consisa Alarcón. Granada.

1993. Junio, Julio. Participa en El Arte de Grabar en Granada. Museo del Grabado Español Contemporáneo. Marbella. Málaga.

1993. Julio. Participa en El Arte de Grabar en Granada. Centro Cultural Puerta de Toledo. Madrid.

1993. Septiembre. Trazos. Galería Espacio D’ de Bubión. Las Alpujarras. Granada.

1994. Noviembre. Doce Grabadores vinculados a Granada. Sala Municipal de Arte. Ceuta.

1995. Abril. Taller Experimental de Grabado Realejo. Centro Cultural Federico García Lorca. Melilla.

1995. Mayo. Taller Experimental de Grabado Realejo. Sala del Instituto de Nador.

1995. Mayo. Taller Experimental de Grabado Realejo. Sala de Arte Municipal. Alhucemas.

1996. Marzo. Taller de Grabado Realejo. Fundación CajaGranada. Sala de Arte. Úbeda. Jaén.

1996. Abril. Taller de Grabado Realejo. Fundación CajaGranada. Sala de Arte. Jaén.

1996. Mayo. Taller de Grabado Realejo. Fundación CajaGranada. Sala Triunfo. Granada.

1996. Junio. Taller de Grabado Realejo. Fundación CajaGranada. Sala de Arte. Motril. Granada.

extranjeras: Santo Domingo, San Juan de Puerto Rico, Kobe, etc.". Lupiáñez, José, op. cit., Pág. 2. 
1996. Noviembre. Stand en la Feria Internacional Estampa 96. Antiguo Museo de Arte Contemporáneo. Madrid.

1997. Mayo. El Realejo según El Realejo. Museo Casa de Los Tiros. Granada.

1998. Mayo, Junio. Retablillo Lorquiano. Museo Casa de Los Tiros. Granada.

1998. Noviembre, Diciembre. Taller Realejo -Exposición-coloquio. Escuela de Arte. Pabellón de Chile. Sevilla.

1999. Mayo. Participa en Antología de Arte Contemporáneo para el Sahara. Palacio de Bibataubín. Granada.

2000. Enero. Participa en Ayuda en Acción. Palacio de La Madraza. Granada.

2000. Junio, Julio. Participa en Estampas para la Paz. Palacio de La Madraza. Granada.

2000. Octubre, Noviembre. Taller de Grabado Realejo. Sala del Ayuntamiento de Las Gabias. Granada.

2001. Mayo, Junio. Taller de Grabado Realejo. Sala del Ayuntamiento de Arjona, Jaén.

2001. Junio, Julio. Participa en Estampas para la Paz. Museo de la Paz. Vall de Uxó, Castellón.

2001. Noviembre, Diciembre. ANDALUZSCIAS. Galería Juanjo Espartero. Sevilla.

2002. Febrero, Marzo. ANDALUZSCIAS. Contemporánea, Centro de Arte. Granada.

2002. Julio. Participa en Estampas para la Paz. Museo Arqueológico. Sevilla.

2003. Octubre. Presentación de la Carpeta "Tiempo de Deseo" dentro de las exposiciones "Los Caminos de Eros". Galería Cartel. Granada.

\section{Museos y Colecciones con obra del Taller de Grabado Realejo.}

El Taller de Grabado Experimental El Realejo colectivamente está representado y se encuentra, entre otras, en las siguientes Colecciones y Museos:

Biblioteca Nacional. Sección de Obra Gráfica Contemporánea. Madrid.

Museo del Grabado Español Contemporáneo. Marbella. Málaga.

Real Academia de Bellas Artes de San Fernando. Calcografía Nacional. Madrid.

Museo de Bellas Artes de Jaén.

Fundación Caja de Granada. Granada.

Museo de El Ermitage. San Petersburgo. Rusia.

Museo Casa de los Tiros. Granada.

Galería Republicana de Arte. Minks. Bielorrusia.

Galería de Arte Moderno. Santo Domingo. República Dominicana.

Consejería de Cultura. Junta de Andalucía. 
Real Academia de Bellas Artes de Granada.

Casa Museo Federico García Lorca. Fuente Vaqueros. Granada

Instituto de la Paz y los Conflictos. Universidad de Granada.

Independientemente, los componentes del Taller han participado en otras colecciones de obra gráfica, y han exhibido su obra en diversas exposiciones, con carácter personal o colectivo, así como están representados en diversas colecciones tanto a nivel nacional como internacional.

\section{BIBLIOGRAFÍA}

AA.VV. Summa Artis. Tomo XI: La Fotografía y el Grabado en la España Contemporánea. Madrid: Editorial Espasa, 2001.

Alcalde, Luisa. El arte de grabar en Granada. Valladolid: Alerta, Enero de 1992.

Calvo, Tomás. "Sobre el Arte, el olvido y la memoria". En la carpeta de grabados El olvido y la memoria. Granada: 1990.

Carrete, Juan; Vega Jesusa. Grabado y Creación Gráfica. Madrid: Historia 16, 1989.

Galera, Pedro. "Grabadores en Granada". Texto del Catálogo de la Caja General. Granada: 1990.

Gómez Segade, J. Manuel. "Obra gráfica del Taller Realejo". Ideal. Granada: Enero de 1987.

Guerrero Villalba, Carmen. Tesis Doctoral Inédita. Arte Gráfico Contemporáneo en Andalucía 1940-1990. Granada: Universidad de Granada, 1997.

Henares Cuéllar, Ignacio. Panorama de las Artes Plásticas en Granada (1950-1990). La renovación de la plástica en Granada ante el 92. Granada: Universidad de Granada, 1992.

Izquierdo, Francisco: Grabadores Granadinos. Madrid. Editorial Marsiega, 1974.

Izquierdo, Francisco. El grabado en Granada. Granada ante el 92. Granada: Universidad de Granada, 1992.

Lupiáñez, José. "El grabado de vanguardia en Granada". Catálogo de la Exposición itinerante Taller de Grabado El Realejo. Granada: Caja General, 1996.

Palma, Carmen. El grabado en Granada durante el siglo XX. Granada: Editorial Universidad de Granada, 1989.

Prieto, Cristina. “Los miembros del Taller Realejo”. Ideal. Granada: abril de 1996.

Vellido, Juan. "Los artistas en torno al grabado. Arte y Letras". Ideal. Granada: Agosto 1991.

Vellido, Juan. "De la Tierra Múltiple". Catálogo AndaluzScias. Taller Experimental de Grabado El Realejo. Granada: Contemporánea Centro de Arte, 2002.

Viribay, Miguel. “El Taller Realejo”. Diario Jaén. Jaén: abril de 1996. 